\title{
Per-site occupancy in the discrete parking problem ${ }^{\text {光 }}$
}

\author{
Seva Shneer ${ }^{\mathrm{a}, 1}$, Peter M. van de Ven ${ }^{\mathrm{b}, *}$ \\ ${ }^{a}$ School of MACS, Department of AMS, Heriot-Watt University, Edinburgh, EH14 4AS, United Kingdom \\ ${ }^{b}$ Centrum Wiskunde 63 Informatica (CWI), P.O. Box 94079, 1090 GB Amsterdam, The Netherlands
}

\begin{abstract}
We consider the classical discrete parking problem, in which cars arrive uniformly at random on any two adjacent sites out of $n$ sites on a line. The car parks on this location if there is no overlap with previously parked cars, and leaves otherwise. This process continues until there is no more space available for cars to park, at which point we may compute the jamming density $E_{n} / n$, which represents the expected fraction of occupied sites. We extend the classical results by not just considering the total expected number of cars parked, but also the probability of each site being occupied by a car. This we then use to provide an alternative derivation of the parking constant.
\end{abstract}

Keywords: generating functions, jamming density, parking problem, wireless networks

\section{Introduction}

Car parking is a classical problem first studied by Rényi [2], where cars of unit length arrive on a line segment uniformly at random. The car parks at this location if and only if there is no overlap with existing cars. This process continues until the configuration of parked cars is such that no new cars can be fitted, at which point we may compute the fraction of the line segment that is utilized for parking, or jamming density. Rényi determined this parking constant as the length of the line segment grows to infinity.

We are interested in the discrete parking problem, which was introduced by Flory [3] and rediscovered by Page [4]. Recall the setting of [4]: there are $n$ sites which form $n-1$ pairs of neighbouring sites. At the first step, a pair $k$ and $k+1$ are chosen at random, and we assume that a car parks in the segment $[k, k+1]$. At the next step, another pair of sites is chosen at random to form a parking spot for the next car but neither site $k$ nor site $k+1$ may be chosen again (as cars cannot overlap). This procedure is performed until there is no pair of neighbouring points that may be chosen. In $[3,4]$ the authors demonstrate that the jamming density grows as $E_{n} / n \rightarrow 1-e^{-2}$ as $n \rightarrow \infty$, with $E_{n}$ the expected number of active sites. Similar results have been obtained for larger cars in $[5,6,7]$. Asymptotic normality of the occupied space was demonstrated in $[8,6]$. The case with multiple rows of parking space is considered by $[9,10]$, and the case of trees in $[11,12]$.

This model has many applications, including polymere chemistry [3, 13], granulometry [14], elections [15], condensation and coagulation [16], genome sequencing [17] and communication networks [18]. We are motivated by the application of resource sharing in communication networks, in particular wireless randomaccess networks. Random-access protocols such as Carrier-Sense Multiple-Access (CSMA) [19] have gained much popularity for their ability to regulate the access of network nodes to a shared medium in a fully distributed fashion, and are for example used in the IEEE 802.11 standard. A node using the CSMA protocol attempts to transmit a packet after some random time, except if any nearby node is already active.

\footnotetext{
Some results appeared without proofs in [1].

* Corresponding author

Email addresses: v.shneer@hw.ac.uk (Seva Shneer), ven@cwi.nl (Peter M. van de Ven)

${ }^{1}$ The research of $\mathrm{S}$. Shneer was supported by EPSRC grant EP/L026767/1
} 
The stochastic process describing the wireless network behaves as follows. We assume that time is slotted, and that each transmission lasts 1 time slot. At the beginning of a time slot, all nodes are inactive, and the time slot is divided into a contention period and a transmission period. During the contention period each node draws a random back-off time, after which it activates as long as no nearby node is already active. This dictates the order in which nodes activate, with the additional constraint that a node may not activate if one of its neighbours is already active.

It is readily seen that these dynamics are identical to that of the parking problem. The application to wireless networks provides us with a useful alternative characterization of the process according to which parking spots are filled, where instead of newly arriving cars selecting a spot uniformly at random, we assume each spot has one dedicated car, and the order in which cars arrive determines the evolution of the parking process. This interpretation was previously used in [20] to provide an alternative approach to determining the parking constant.

Previous studies of the parking problem have focused on metrics such as the jamming density or the distribution of the gap sizes. Instead, we are interested in the probability that each site is occupied, both in the case of a finite $n$ and the asymptotic regime. This is motivated by the application to wireless networks, where the probability of a site being occupied is equivalent to the throughput of the wireless transmitter located on that site.

We start the next section by providing a rigorous definition of the activation process in a network governed by the CSMA protocol and explain its equivalence to the classical parking problem. We then proceed to derive the probability that a given node of a network of $n$ nodes is active, which is the main result of the paper. We then formulate two corollaries, including a derivation of the expected number of the active nodes in a network of length $n$, which is equal to the expected number of cars that can park on a line segment of length $n-1$.

\section{Model and result}

Consider a linear network of $n$ sites numbered $1, \ldots, n$. We draw a random permutation of sites $\sigma(1), \ldots, \sigma(n)$, and sites attempt to activate in this order. Such an attempt is successful if neither of the site's neighbours is already active. The set of sites active at the end of this process is fully determined by the permutation, and the end configuration is such that the gap between two active sites in at most 2, i.e., no additional sites can be activated. The relation to the classical parking problem is immediate: the site with the highest priority order may be considered to be the left one of the pair of sites (out of sites $1,2, \ldots, n+1$ ) chosen first. Subsequent steps are also equivalent.

It is worth mentioning that in both the classical parking problem and the setting of our note, one can consider cars (or interference regions) of length bigger than 1 and our technique may be applied in this general setting. However, we limit ourselves to the case of length 1 for ease of presentation.

We are interested in the probability of a site being active. This quantity corresponds to the per-node throughputs in a wireless network. We denote by $T_{i}(n)$ the probability that node $i$ is active. If one is interested in the probability $S_{i}(n)$ that site $i$ is occupied in the classical setting, then clearly,

$$
S_{i}(n)=T_{i}(n-1)+T_{i-1}(n-1)
$$

with the convention that $T_{-1}(k)=0$ for all $k$.

The next theorem is our main result.

Theorem 1. For $n \geq 1$ and $1 \leq i \leq n$,

$$
T_{i}(n)= \begin{cases}1+\sum_{k=0}^{\frac{n-i}{2}} d_{i, i+2 k}, & \text { if }(n-i) \text { is even, } \\ \frac{n-i-1}{\sum_{k=0}^{2}} d_{i, i+2 k+1}, & \text { if }(n-i) \text { is odd }\end{cases}
$$


where

$$
d_{i, i+l}=\sum_{k=0}^{i-1} \frac{(-1)^{l+k}}{k !}\left(\frac{1}{(i+l-k) !}-\frac{1}{l !}\right)
$$

Proof. Conditioning on the first node to activate yields the following recursive equation:

$$
T_{i}(n)=\frac{1}{n}+\frac{1}{n} \sum_{j=1}^{i-2} T_{i-j-1}(n-j-1)+\frac{1}{n} \sum_{j=i}^{n-2} T_{i}(j) .
$$

With $\psi_{i}(\rho)=\sum_{n=i}^{\infty} T_{i}(n) \rho^{n}$, summing (3) over $n$ gives the differential equation

$$
\psi_{i}^{\prime}(\rho)=\sum_{j=1}^{i-2} \rho^{j} \psi_{i-j-1}(\rho)+\frac{\rho^{i-1}}{1-\rho}+\frac{\rho}{1-\rho} \psi_{i}(\rho),
$$

with initial condition $\psi_{i}(0)=0$.

We shall show below that

$$
\psi_{i}(\rho)=\frac{1}{1-\rho^{2}}\left(\rho^{i}+(-1)^{i+1}-e^{-\rho} \rho^{i} a_{i}+(-1)^{i} e^{-\rho} \sum_{k=0}^{i-1} \frac{\rho^{k}}{k !}\right),
$$

which leaves (1) to prove. To this end we shall find the Taylor expansion for (5) with respect to the powers of $\rho$. Let us start with the last term inside the brackets in (5):

$$
e^{-\rho} \sum_{k=0}^{i-1} \frac{\rho^{k}}{k !}=\sum_{m=0}^{\infty} \frac{(-1)^{m} \rho^{m}}{m !} \cdot \sum_{k=0}^{i-1} \frac{\rho^{k}}{k !}=\sum_{m=0}^{\infty} c_{m} \rho^{m}
$$

with

$$
c_{m}=\left\{\begin{array}{l}
1, \quad \text { if } \quad m=0, \\
\sum_{k=0}^{n} \frac{(-1)^{m-k}}{k !(m-k) !}=0, \quad \text { if } \quad 0<m \leq i-1, \\
\sum_{k=0}^{i-1} \frac{(-1)^{m-k}}{k !(m-k) !}=b_{i, m}, \quad \text { if } \quad m \geq i,
\end{array}\right.
$$

and

$$
b_{i, n}=\sum_{k=0}^{i-1} \frac{(-1)^{n-k}}{k !(n-k) !} .
$$

Substituting this into (5) and using the Taylor expansion for the exponential function

$$
e^{-\rho s}=\sum_{m=0}^{\infty}(-1)^{m} \frac{\rho^{m}}{m !} s^{m}
$$

yields

$$
\begin{aligned}
\psi_{i}(\rho) & =\frac{1}{1-\rho^{2}}\left(\rho^{i}+a_{i} \sum_{k=0}^{i-1} \frac{(-1)^{k}}{k !} \sum_{m=i}^{\infty} \frac{(-1)^{m-i}}{(m-i) !} \rho^{m}+(-1)^{i} \sum_{m=i}^{\infty} b_{i, m} \rho^{m}\right) \\
& =\frac{1}{1-\rho^{2}}\left(\rho^{i}+\sum_{m=i}^{\infty} d_{i, m} \rho^{m}\right)
\end{aligned}
$$

with $d_{i, m}$ defined in (2). The $T_{i}(n)$ then readily follow from $\psi_{i}(\rho)$. 
This proves Theorem 1. It remains to be shown that (5) holds. Introducing

$$
\nu(\rho, s)=\sum_{i=1}^{\infty} \psi_{i}(\rho) s^{i}
$$

and using (4) gives

$$
\begin{aligned}
\frac{\partial \nu(\rho, s)}{\partial \rho} & =\sum_{i=1}^{\infty} \psi_{i}^{\prime}(\rho) s^{i}=\sum_{i=1}^{\infty} \sum_{j=1}^{i-2} \rho^{j} \psi_{i-j-1}(\rho) s^{i}+\sum_{i=1}^{\infty} \frac{\rho^{i-1} s^{i}}{1-\rho}+\sum_{i=1}^{\infty} \frac{\rho}{1-\rho} \psi_{i}(\rho) s^{i} \\
& =\sum_{j=1}^{\infty} \rho^{j} \sum_{i=j+2}^{\infty} \psi_{i-j-1}(\rho) s^{i}+\frac{s}{(1-\rho)(1-\rho s)}+\frac{\rho}{1-\rho} \nu(\rho, s) \\
& =\left(\frac{\rho s^{2}}{1-\rho s}+\frac{\rho}{1-\rho}\right) \nu(\rho, s)+\frac{s}{(1-\rho)(1-\rho s)},
\end{aligned}
$$

and $\nu(0, s)=0$. Solving this standard differential equation we obtain

$$
\nu(\rho, s)=\frac{\left.s\left(1-e^{-\rho(s+1)}\right)\right)}{(s+1)(1-\rho)(1-\rho s)} .
$$

We now need to write the Taylor expansion for the latter expression. Using

$$
\frac{s}{s+1}=\sum_{m=1}^{\infty}(-1)^{m+1} s^{m} \quad \text { and } \quad \frac{1}{1-\rho s}=\sum_{k=0}^{\infty} \rho^{k} s^{k}
$$

yields

$$
\begin{aligned}
\frac{s}{s+1} \frac{1}{1-\rho s} & =\sum_{l=1}^{\infty}\left(\sum_{k=0}^{l-1} \rho^{k}(-1)^{l-k+1}\right) s^{l}=\sum_{l=1}^{\infty}(-1)^{l+1}\left(\sum_{k=0}^{l-1} \rho^{k}(-1)^{-k}\right) s^{l} \\
& =\sum_{l=1}^{\infty}(-1)^{l+1} \frac{1-(-\rho)^{l}}{1+\rho} s^{l}=\sum_{l=1}^{\infty} \frac{\rho^{l}+(-1)^{l+1}}{1+\rho} s^{l} .
\end{aligned}
$$

Substituting (9) and (7) into (8) gives

$$
\begin{aligned}
& \nu(\rho, s) \\
= & \frac{s}{s+1} \frac{1}{1-\rho} \frac{1}{1-\rho s}\left(1-e^{-\rho(s+1)}\right)=\frac{1}{1-\rho^{2}}\left(\sum_{m=1}^{\infty}\left(\rho^{m}+(-1)^{m+1}\right) s^{m} \cdot\left(1-e^{-\rho(s+1)}\right)\right) \\
= & \frac{1}{1-\rho^{2}}\left(\sum_{m=1}^{\infty}\left(\rho^{m}+(-1)^{m}\right) s^{m}-e^{-\rho} \sum_{m=1}^{\infty} \rho^{m} s^{m} \sum_{k=0}^{m-1} \frac{(-1)^{k}}{k !}+e^{-\rho} \sum_{m=1}^{\infty}(-1)^{m} s^{m} \sum_{k=0}^{m-1} \frac{\rho^{k}}{k !}\right),
\end{aligned}
$$

which yields (5).

Theorem 1 provides us with a closed-form but unwieldy expression for the individual throughputs. In case the network size grows to infinity we can obtain a more elegant expression for the throughputs of nodes 1 and 2 .

Corollary 1. As $n \rightarrow \infty$,

$$
T_{1}(n) \rightarrow 1-\mathrm{e}^{-1} \text { and } T_{2}(n) \rightarrow \mathrm{e}^{-1} .
$$

From Theorem 1 we can recover $E_{n}$ and the jamming density $E_{n} / n$, defined as the expected number of occupied sites in the classical parking problem. To do this, observe that due to boundary effects, $E_{n}=2 F_{n-1}$, where $F_{n-1}$ represents the expected number of active nodes in an $n-1$ node wireless network. By adding the individual activation probabilities we obtain the following result. 
Corollary 2. The total expected number of active nodes is given as

$$
F_{n}=\sum_{k=1}^{n}(-1)^{k+1} \frac{2^{k-1}}{k !}(n-k+1), \quad n=1,2, \ldots
$$

Before proceeding to the proof, note that it is straightforward to check that

$$
\lim _{n \rightarrow \infty} \frac{F_{n}}{n}=\frac{1}{2}\left(1-e^{-2}\right)=\frac{1}{2} \lim _{n \rightarrow \infty} \frac{E_{n}}{n},
$$

and we recover the result of $[3,4]$.

Let us now prove the corollary.

Proof. Let $n$ be even; the proof for odd $n$ is analogous. By summing over the individual throughputs from (1), the total throughput may be written as

$$
F_{n}=\sum_{i=1}^{n} T_{i}(n)=\frac{n}{2}+\sum_{j=1}^{n / 2} \sum_{k=0}^{n-2 j+1} \sum_{l=0}^{2 j-1} \frac{(-1)^{k+l+1}}{k ! l !}=: \frac{n}{2}+A
$$

where $A$ is defined as the second part of (10). In order to rewrite $A$, let

$$
B:=\sum_{j=1}^{n / 2} \sum_{k=0}^{n-2 j} \sum_{l=0}^{2 j} \frac{(-1)^{k+l+1}}{k ! l !} .
$$

We may rewrite $B$ as

$$
\begin{aligned}
B & =\sum_{j=1}^{n / 2} \sum_{l=0}^{2 j-1} \sum_{k=0}^{n-2 j+1} \frac{(-1)^{k+l+1}}{k ! l !}-\sum_{j=1}^{n / 2} \sum_{l=0}^{2 j-1} \frac{(-1)^{n-2 j+2+l}}{(n-2 j+1) ! l !}+\sum_{j=1}^{n / 2} \sum_{k=0}^{n-2 j} \frac{(-1)^{k+2 j+1}}{k !(2 j) !} \\
& =A-\sum_{k=1}^{n / 2} \sum_{l=0}^{n-2 k+1} \frac{(-1)^{l}}{(2 k-1) ! l !}+\sum_{j=1}^{n / 2} \sum_{k=0}^{n-2 j} \frac{(-1)^{k+2 j+1}}{k !(2 j) !} \\
& =A+\sum_{i=1}^{n} \sum_{k=0}^{n-i} \frac{(-1)^{k+1}}{k ! i !}
\end{aligned}
$$

where the second equality is due to the transformation $k=n / 2-j+1$. Next, we may write

$$
\begin{aligned}
\sum_{i=1}^{n} \sum_{k=0}^{n-i} \frac{(-1)^{k+1}}{k ! i !} & =\sum_{i=0}^{n} \sum_{k=0}^{n-i} \frac{(-1)^{k+1}}{k ! i !}-\sum_{k=0}^{n} \frac{(-1)^{k+1}}{k !} \\
& =\sum_{t=0}^{n}(-1)^{t+1} \sum_{l=0}^{t} \frac{(-1)^{l}}{(t-l) ! l !}-\sum_{k=0}^{n} \frac{(-1)^{k+1}}{k !} \\
& =-1+\sum_{t=1}^{n}(-1)^{t+1}(1+(-1))^{t} t !-\sum_{k=0}^{n} \frac{(-1)^{k+1}}{k !}=-1-\sum_{k=0}^{n} \frac{(-1)^{k+1}}{k !} .
\end{aligned}
$$

Substituting this into (11), and using the identity

$$
A+B=\sum_{i=1}^{n} \sum_{k=0}^{n-i} \sum_{l=0}^{i} \frac{(-1)^{k+l+1}}{k ! l !}
$$


we obtain

$$
A=\frac{1}{2}\left(\sum_{i=1}^{n} \sum_{k=0}^{n-i} \sum_{l=0}^{i} \frac{(-1)^{k+l+1}}{k ! l !}+\sum_{k=0}^{n} \frac{(-1)^{k+1}}{k !}+1\right)=\frac{1}{2} \sum_{i=0}^{n} \sum_{k=0}^{n-i} \sum_{l=0}^{i} \frac{(-1)^{k+l+1}}{k ! l !}+\frac{1}{2} .
$$

By rearranging the order of summation, and substituting $t=k+l$, we obtain

$$
A=\frac{1}{2}+\frac{1}{2} \sum_{l=0}^{n} \sum_{k=0}^{n-l}(n-k-l+1) \frac{(-1)^{k+l+1}}{k ! l !}=\frac{1}{2}+\frac{1}{2} \sum_{l=0}^{n} \sum_{t=l}^{n}(n-t+1) \frac{(-1)^{t+1}}{l !(t-l) !} .
$$

If we interchange summation, we can apply the binomial theorem to arrive at

$$
\begin{aligned}
A & =\frac{1}{2}+\frac{1}{2} \sum_{t=0}^{n}(-1)^{t+1} \frac{(n-t+1)}{t !} \sum_{l=0}^{t} \frac{t !}{l !(t-l) !} \\
& =\frac{1}{2}+\frac{1}{2} \sum_{t=0}^{n}(-1)^{t+1} \frac{2^{t}}{t !}(n-t+1)=\frac{1}{2}-\frac{n+1}{2}+\sum_{t=1}^{n}(-1)^{t+1} \frac{2^{t-1}}{t !}(n-t+1) .
\end{aligned}
$$

Substituting this into (10), we obtain the desired result.

\section{References}

[1] S. Shneer, P. M. van de Ven, Comparing slotted and continuous CSMA: throughputs and fairness, in: In proc. IFIP Performance, Vol. 39, 2011, pp. 35-37.

[2] A. Rényi, Car parking problem, Sel. Trans. Math. Stat. Prob 4 (1963) 205.

[3] P. Flory, Intramolecular reaction between neighboring substituents of vinyl polymers, Journal of the American Chemical Society 61 (6) (1939) 1518-1521.

[4] E. Page, The distribution of vacancies on a line, Journal of the Royal Statistical Society. Series B (Methodological) (1959) 364-374.

[5] H. D. Friedman, D. Rothman, J. K. MacKenzie, Problem 62-3, SIAM Review 6 (2) (1964) 180-182.

[6] C. A. J. Klaassen, J. T. Runnenburg, Discrete spacings, Statistica neerlandica 57 (4) (2003) $470-483$.

[7] R. G. Pinsky, A one-dimensional probabilistic packing problem, in: Problems from the Discrete to the Continuous, 2014, pp. $21-34$.

[8] J. T. Runnenburg, Asymptotic normality in vacancies on a line, Statistica Neerlandica 36 (3) (1982) $135-148$.

[9] K. Georgiou, E. Kranakis, D. Krizanc, Random maximal independent sets and the unfriendly theater seating arrangement problem, Discrete Mathematics 309 (16) (2009) 5120-5129.

[10] H.-H. Chern, H.-K. Hwang, T.-H. Tsai, Random unfriendly seating arrangement in a dining table, Advances in Applied Mathematics 65 (2015) 38-64.

[11] M. D. Penrose, A. Sudbury, Exact and approximate results for deposition and annihilation processes on graphs, The Annals of Applied Probability 15 (1B) (2005) 853-889.

[12] H. G. Dehling, S. R. Fleurke, C. Külske, Parking on a random tree, Journal of Statistical Physics 133 (1) (2008) $151-157$.

[13] J. K. Mackenzie, Sequential filling of a line by intervals placed at random and its application to linear adsorption, The Journal of Chemical Physics 37 (4) (1962) 723-728.

[14] K. Gotoh, W. S. Jodrey, E. M. Tory, Average nearest-neighbour spacing in a random dispersion of equal spheres, Powder Technology 21 (2) (1978) 285-287.

[15] Y. Itoh, S. Ueda, A random packing model for elections, Annals of the Institute of Statistical Mathematics 31 (1) (1979) $157-167$

[16] D. W. Cooper, Parking problem (sequential packing) simulations in two and three dimensions, Journal of colloid and interface science 119 (2) (1987) 442-450.

[17] J. C. Roach, V. Thorsson, A. F. Siegel, Parking strategies for genome sequencing, Genome research 10 (7) (2000) 10201030.

[18] E. G. Coffman Jr, L. Flatto, P. Jelenković, B. Poonen, Packing random intervals on-line, Algorithmica 22 (4) (1998) $448-476$.

[19] L. Kleinrock, F. A. Tobagi, Packet switching in radio channels: part I - carrier sense multiple-access modes and their throughput-delay characteristics, IEEE Trans. Commun. 23 (12) (1975) 1400-1416.

[20] L. Gerin, The Page-Rényi parking process, arXiv preprint arXiv:1411.8002. 